\title{
THE CONJUGACY PROBLEM FOR BOUNDARY LOOPS IN 3-MANIFOLDS
}

BY

BENNY D. EVANS ${ }^{1}$

\begin{abstract}
A geometric solution of the word problem for fundamental groups of compact, orientable, irreducible, sufficiently large 3-manifolds has been given by $\mathrm{F}$. Waldhausen. We present here a solution of a restricted version of the conjugacy problem for this same class of 3-manifolds; however, the conjugacy problem for 3-manifolds remains in general unsolved. The main results is that there is an algorithm that will determine for any two loops $L_{1}, L_{2}$ in the boundary of a compact, orientable, irreducible sufficiently large 3-manifold $M$ if $L_{1}$ is freely homotopic in $M$ to $L_{2}$.
\end{abstract}

1. Waldhausen's results on the word problem [6] are used in proving Theorem 3.2; however, the techniques used for solving the word problem are not generally effective in studying free homotopies. In particular, it is difficult to detect the existence of free homotpies in the presence of high genus cutting surfaces. This problem can be avoided in the case of boundary loops, thanks to a second theorem of Waldhausen [7], [2] which assures us that if an essential disk or annulus does not exist in a 3-manifold $M$, then all free homotopies of boundary loops in $M$ occur in the boundary of $M$.

In order to prove Theorem 3.2, a second algorithm is produced that is of interest. If $l$ is a loop in a 2-manifold $F$, we use the notation $(l, F)$ to denote the collection of all loops in $F$ that are freely homotopic in $F$ to $l$.

THEOREM. There is an algorithm BA that will construct for any loop $l$ in the boundary of a compact, orientable, irreducible 3-manifold $M$ and for any incompressible subset $F$ of the boundary of $M$ one representative from each class $(k, F)$ such that $l$ is freely homotopic in $M$ to a loop in $(k, F)$.

Implicit in the statement that $B A$ is an algorithm is the fact that there are only finitely many classes $(k, F)$ whose members are freely homotopic to a fixed loop $l$. Thus as a corollary we obtain the following result of W. Jaco [5].

THEOREM (W. JACO). Let $F$ be an incompressible subset of the boundary of a 3-manifold $M$. If $l$ is a loop in $M$ then there are only finitely many classes $(k, F)$

Received by the editors October 8, 1974 and, in revised form, June 4, 1976.

AMS (MOS) subject classifications (1970). Primary 20F10, 57A10.

Key words and phrases. Free homotopy, sufficiently large 3-manifold, conjugacy problem.

${ }^{1}$ Research partially supported by National Science Foundation grant GP-38819. 
such that $l$ is freely homotopic in $M$ to a member of $(k, F)$.

2. Preparations. For definitions of such terms as incompressible 2-manifold, boundary incompressible 2-manifold, irreducible 3-manifold, and sufficiently large 3-manifold, the reader is referred to [8]. A disk $D$ properly embedded in a 3-manifold $M$ is essential in $M$ if bd $D$ is not contractible in bd $M$. An annulus properly embedded in a 3-manifold $M$ is essential if it incompressible and boundary incompressible in $M$. A cutting surface for a 3-manifold $M$ is an incompressible, boundary incompressible 2-manifold $F$ properly embedded in $M$ such that $\mathrm{bd} F=\varnothing$ if and only if bd $M=\varnothing$. If $F$ is a 2-manifold properly embedded in $M$, we shall refer to the 3-manifold that is the closure of the complement of a regular neighborhood of $F$ in $M$ as $M$ cut at $F$. An arc is an embedding of the unit interval. A path is an immersion of the unit interval.

The first step is to produce an algorithm that will construct special types of cutting surfaces. The importance of this procedure will become apparent as the paper develops. The following algorithm of W. Haken [4] will be useful.

2.1 TheOREM (HAKeN). Let $M$ be a compact, orientable, irreducible 3-manifold and $J$ a finite graph in bd $M$. For any value of $n$, there is an algorithm $H$ for finding out if there exists in $M$ an essential disk meeting $J$ in fewer than $n$ points. If such a disk exists, the algorithm will find one.

A collection of disks $C=\left\{D_{1}, \ldots, D_{r}\right\}$ properly embedded in a compact, orientable, irreducible 3-manifold $M$ is a complete set of cutting disks for $M$ if:

(i) For $i \neq j, D_{i} \cap D_{j}=\varnothing$.

(ii) $D_{j}$ is essential in $M$ cut at $\cup_{i \neq j} D_{i}$.

(iii) No component of $M$ cut at $C$ contains an essential disk.

Let $F$ denote an incompressible submanifold of bd $M$ and $C$ a complete set of cutting disks for $M$. Then $C$ is irreducible with respect to $F$ if whenever a path $l$ in $C$ is homotopic with endpoints fixed to a path $k$ in $F$, then $l$ is homotopic with endpoints fixed to $k$ in $F \cup C$.

2.2 LEMMA. Let $M$ denote a compact, orientable, irreducible 3-manifold and $F$ an incompressible submanifold of bd $M$. There is an algorithm that will construct a complete set $C$ of cutting disks for $M$ that is irreducible with respect to $F$.

Proof. Haken's algorithm $H$ [4] can be applied to determine if there is an essential disk in $M$. If no such disk exists, put $C=\varnothing$; otherwise, the algorithm will produce one such disk $D_{1}$. If an essential disk exists in $M$ cut at $D_{1}$, Haken's algorithm will produce a disk $D_{2}$ that meets bd $D_{1}$ minimally. Clearly if any such disk exists, there is one that misses $D_{1}$ altogether. Hence $D_{1} \cap D_{2}=\varnothing$. Haken's algorithm is applied repeatedly in this fashion to 
construct a mutually exclusive collection $C+=\left\{D_{1}, \ldots, D_{s}\right\}$ of cutting disks for $M$ with the property that $M$ cut at $C+$ contains no essential disks. (Certainly this stage must be reached in no more than 2 rank $\pi_{1}(M)$ steps.)

At this point, it may be so that some $D_{j}$ is inessential in $M$ cut at $\cup_{i \neq j} D_{i}$. (It is, for example, possible via the above construction to arrive at five cutting disks for a genus three cube-with-handles.) If this is the case, then $D_{j}$ cobounds a 3-cell in $M$ cut at $\cup_{i \neq j} D_{i}$ with a disk $E$ in the boundary of $M$ cut at $\cup_{i \neq j} D_{i}$. It follows that each component of $M$ cut at $\cup_{i \neq j} D_{i}$ is homeomorphic with a component of $M$ cut at $C+$ so that no component of $M$ cut at $\cup_{i \neq j} D_{i}$ contains an essential disk. Thus $D_{j}$ can be deleted from the collection $C+$ without sacrificing property (iii) in the definition of a complete set of cutting disks. After deleting all such extra disks from $C+$, the remaining collection $C=\left\{D_{1}, \ldots, D_{r}\right\}$ is clearly a complete set of cutting disks for $M$.

The complexity of a complete set of cutting disks $C$ (denoted by $\# C$ ) is the total number of points in the intersection $C \cap$ bd $F$. Let $K$ denote the boundary of $F \cap\left(M\right.$ cut at $C$ ). If $\alpha$ is an arc in bd $D_{j}$ that is homotopic in $M$ cut at $C$ with endpoints fixed to an $\operatorname{arc} \beta$ in $K$, and if $\alpha$ is not homotopic in $F \cup D_{j}$ to $\beta$, then we shall describe an operation $\Sigma$ that will produce a new complete set of cutting disks $C^{*}$ with $\# C^{*}<\# C$. The idea is quite simple. Its description is unfortunately a bit awkward. Note first of all that the incompressibility of $F$ assures us that (int $\alpha) \cap(\mathrm{bd} F) \neq \varnothing$. Let $L$ denote the component of $M$ cut at $C$ that contains $\beta$. Since bd $L$ is incompressible, the loop theorem assures us that $\alpha$ is homotopic to $\beta$ in $\operatorname{bd} L$ and hence $\alpha$ cobounds a disk $E$ with $\beta$ in bd $L$. If $E$ contains $D_{j}$, then note that $\operatorname{cl}\left(\left(\right.\right.$ bd $\left.\left.D_{j}\right)-\alpha\right)$ is not homotopic in $F \cup D_{j}$ to $\beta$ since $\alpha$ is homotopic in $D_{j}$ to $\operatorname{cl}\left(\operatorname{bd}\left(D_{j}\right)-\alpha\right)$. So, if $D_{j} \subset E$, replace $\alpha$ in what follows by $\operatorname{cl}\left(\left(\operatorname{bd} D_{j}\right)-\alpha\right)$ and replace $E$ by $\operatorname{cl}\left(E-D_{j}\right)$. We proceed assuming $E \cap D_{j}=\alpha$. By a small deformation of $E$, we obtain a disk $E^{*}$ with the following properties.

(i) bd $E^{*}=\alpha^{*} \cup \beta^{*}$ is freely homotopic in bl $L$ with bd $E$.

(ii) int $E^{*} \subset$ int $L$.

(iii) int $\alpha^{*} \subset$ int $D_{j}$.

(iv) $\beta^{*} \subset F$.

The disk $E^{*}$ cobounds a 3-cell $R$ in $L$ with a disk in the boundary of $L$. The arc $\alpha^{*}$ separates $D_{j}$ into two disks $P \subset R$ and $Q$. Put $D_{i}^{*}=D_{i}$ if $i \neq j$, $D_{j}^{*}=Q \cup E^{*}$, and $C^{*}=\left\{D_{1}^{*}, D_{2}^{*}, \ldots, D_{r}^{*}\right\}$. This alteration has the effect of replacing $\alpha$ in bd $C$ by $\beta^{*}$ so that since $\alpha \cap$ bd $F \neq \varnothing$ and $\beta^{*} \subset F$, it is clear that $\# C^{*}<\# C$.

It must be shown that $C^{*}$ is again a complete set of cutting disks for $M$. To this end, let $L_{1}$ denote a second component of $M$ cut at $C$ that meets $D_{j}$. (Possibly $L_{1}=L$.) Observe first of all that $M$ cut at $C^{*}$ is obtained from $M$ 
cut at $C$ by cutting away the 3-cell $R$ from $L$ along $E^{*}$ and attaching $R$ to $L_{1}$ along the disk $P$. In particular, each component of $M$ cut at $C^{*}$ is homeomorphic with a component of $M$ cut at $C$ so that no component of $M$ cut at $C^{*}$ contains an essential disk. Also, $D_{j}^{*}$ is isotopic to $D_{j}$ in $M$ cut at $\cup_{i \neq j} D_{i}$ and so is essential in $M$ cut at $\cup_{i \neq j} D_{i}^{*}$. Suppose $k \neq j$, and bd $D_{k}^{*}$ bounds a disk $S$ in the boundary of $M$ cut at $\cup_{i \neq k} D_{i}^{*}$. Then $S$ must contain $D_{j}^{*}$ in its interior; otherwise, $D_{k}$ is inessential in $M$ cut at $\cup_{i \neq k} D_{i}$. The disk $S$ meets the 3-cell $R$ along $E^{*}$. But then $E^{*}$ can be replaced by its complement in bd $R$ to produce a disk $S^{*}$ in the boundary of $M$ cut at $\cup_{i \neq k} D_{i}$ whose boundary is bd $D_{k}$. This is, of course, not consistent with the fact that $C$ is a complete set of cutting disks for $M$.

In view of the fact that the word problem is solvable for 2-manifolds [1], it is possible to determine if any arc $\alpha$ in bd $C$ is homotopic in $M$ cut at $C$ with endpoints fixed to an $\operatorname{arc} \beta$ in $K$, but $\alpha$ is not homotopic to $\beta$ in $F \cup C$. Then as shown above, the operation $\Sigma$ can be applied to $C$ to produce a new complete set of cutting disks $C^{*}$ with $\# C^{*}<\# C$. As $\# C$ is finite, the above algorithm can be applied repeatedly to produce a complete set of cutting disks (which we again call $C$ ) with the following property.

(A) If $\alpha$ is any arc in bd $C$ that is homotopic in $M$ cut at $C$ with endpoints fixed to an arc $\beta$ in $K$, then $\alpha$ is homotopic to $\beta$ in $F \cup C$.

It remains to show that $C$ is irreducible with respect to $F$. In order to avoid a mass of notation, we shall accomplish this in three steps. We establish first the property:

(A1) If $\alpha$ is any arc in bd $C$ that is homotopic in the boundary of $M$ cut at $C$ with endpoints fixed to a path $k$ in $F$, then $\alpha$ is homotopic with endpoints fixed to $k$ in $F \cup C$.

Suppose then that $f: E \rightarrow M$ is a homotopy satisfying the hypothesis of (A1). ( $E$ is a 2-cell, bd $E$ consists of two arcs $\sigma, \lambda$ with $f(\sigma)=\alpha, f(\lambda)=k$.) The map $f$ is taken to be transverse with respect to bd $F$ and among all such homotopies of this type which cannot be deformed into $F, f$ is chosen so that the number of components of $f^{-1}(\mathrm{bd} F)$ is a minimum. The incompressibility of $F$ assures us then that $f^{-1}(\mathrm{bd} F)$ consists of spanning arcs. Let $E_{1}$ denote the closure of the component of $E-f^{-1}(\mathrm{bd} F)$ that contains $\lambda$, and let $\tau$ denote the component of $f^{-1}(\mathrm{bd} F)$ that lies in the boundary of $E_{1}$ and has its initial point adjacent to the initial point of $\sigma$. The endpoints of $\tau$ divide $\sigma$ into three arcs $\sigma_{1}, \sigma_{2}, \sigma_{3} . \sigma_{1}$ has the same initial points as $\sigma$, the endpoints of $\sigma_{2}$ coincide with those of $\tau$, and the terminal point of $\sigma_{3}$ is the terminal point of $\sigma$. The arc $\tau$ cobounds a disk $E_{2}$ with $\sigma_{2}$. Let $E_{3}$ denote the closure of $E-E_{2}, f_{2}=f\left|E_{2}, f_{3}=f\right| E_{3}$. Making use of property (A) of the collection $C$, it is possible to deform $f_{2}$ into $F \cup C$. The map $f_{3}$ can be regarded as a homotopy of the path $f\left(\tau^{-1}\right) f\left(\sigma_{1}^{-1}\right) k \subset F$ to the arc $f\left(\sigma_{3}\right) \subset$ bd $C$. Furthermore, after a small deformation of $f_{3}$ to make it transverse with respect to 
bd $F$ the number of components of $f_{3}^{-1}(\mathrm{bd} F)$ is smaller than the number of components of $f^{-1}(\mathrm{bd} F)$. The minimality assumption for $f$ then insures that $f_{3}$ can also be deformed into $F \cup C$. This establishes property (Al) for $C$.

(A2) If $\alpha$ is any path in $D_{j} \subset C$ that is homotopic in $M$ cut at $C$ with endpoints fixed to a path $k$ in $F$, then $\alpha$ is homotopic with endpoints fixed to $k$ in $F \cup C$.

Let $f: E \rightarrow M$ be a homotopy satisfying the hypothesis of (A2). Since $M$ cut at $C$ has incompressible boundary, the map $f$ can be deformed into the boundary of $M$ cut at $C$. This deformation can clearly be chosen so that $f^{-1}\left(\mathrm{bd} D_{j}\right)$ is a single spanning arc $\tau$ in $E$. Then $\tau$ separates $E$ into two disks $E_{1}\left(f\left(E_{1}\right) \subset D_{j}\right)$ and $E_{2}$. Observe that $f \mid E_{2}$ satisfies the hypothesis of property (A1) so that it can be deformed into $F \cup C$.

Finally we show that $C$ is irreducible with respect to $F$. Let $f: E \rightarrow M$ denote a homotopy where bd $E$ consists of two arcs $\alpha, \beta$ with $f(\alpha) \subset C$ and $f(\beta) \subset F$. The map $f$ is taken to be transverse with respect to $C$, and among all such homotopies that cannot be deformed into $F \cup C, f$ is chosen so that the number of components of $f^{-1}(C)$ is minimal. It is then immediate that no component of $f^{-1}(C)$ is a simple closed curve. Let $\tau$ denote an innermost spanning arc in $f^{-1}(C)$. The endpoints of $\tau$ divide $\beta$ into three arcs $\beta_{1}, \beta_{2}, \beta_{3}$ where the endpoints of $\beta_{2}$ coincide with those of $\tau$. Now $\tau$ divides $E$ into two disks $E_{1}$ containing $\beta_{2}$ and $E_{2}$. Put $f_{1}=f \mid E_{1}$ and $f_{2}=f \mid E_{2}$. Property (A2) is available to deform $f_{1}$ into $F \cup D_{j} .\left(f(\tau) \subset D_{j}.\right)$ Thus $f(\tau)$ is homotopic to an arc in the boundary of $D_{j}$ that is contained in $F$. Regarding $f_{2}$ as a homotopy of $f(\alpha) \subset C$ to $f\left(\beta_{1} \tau \beta_{2}\right) \subset F$, the minimality assumption on $f$ allows $f_{2}$ to be deformed into $F \cup C$. This completes the proof of Lemma 2.2.

$S^{1}$ shall denote the unit sphere in the complex plane with the real number 1 as base point. If $F$ is a 2-manifold properly embedded in $M$, and $l$ a loop in $M$ meeting $F$ at a point $P$, then $l$ meets $F$ essentially at $P$ if given any map $f$ : $S^{1} \times[0,1] \rightarrow M$ such that $f(1,0)=P, f \mid S^{1} \times 0=l$, and $f$ is transverse with respect to $F$, then the component of $f^{-1}(K)$ meeting $(1,0)$ is an arc joining $S^{1} \times 0$ with $S^{1} \times 1$. A loop meets a 2 -manifold essentially if it meets it essentially at some point.

If $l$ is a loop in a 3-manifold $M$ and $F$ an incompressible subset of the boundary of $M$, then there are possibly many free homotopies of $l$ into $F$. Our next goal is to show that in order to find all classes $(k, F)$ that are freely homotopic in $M$ to $l$, it suffices to consider special types of homotopies. Let $K$ denote an essential disk or annulus for $M$. Let $f: S^{1} \times[0,1] \rightarrow M$ be a map such that $f \mid S^{1} \times 0=l$, and suppose $f$ is transverse with respect to $K$. The type of the homotopy $f$ with respect to $K$ is determined according to the following criteria.

Type $0 . f^{\cdot-1}(K)=\varnothing$. 
Type 1. $f^{-1}(K)$ consists of a single nontrivial simple closed curve on $S^{1} \times[0,1]$.

Type 2. $f^{-1}(K)$ consists of two nontrivial curves $k_{1}, k_{2}$, and $f\left(k_{1}\right)$ is homotopic on $K$ to $f\left(k_{2}\right)^{-1}$.

Type 3. $l$ meets $K$ essentially.

2.3 LEMMA. Let $l$ be a noncontractible loop in the boundary of a compact, orientable, irreducible 3-manifold $M, F$ an incompressible submanifold of bd $M$, and $k$ a loop in $F$ that is freely homotopic in $M$ to $l$. Suppose $C$ is a complete set of cutting disks for $M$ that is irreducible with respect to $F$ and that $l \cap C=\varnothing$. Then there is a loop $k_{1} \in(k, F)$ and a type 0 homotopy with respect to $C$ of $l$ to $k_{1}$.

Proof. Among all elements of $(k, F)$ that are freely homotopic in $M$ to $l$, choose $k_{1}$ so that the number of points in $k_{1} \cap C$ is minimal. Let $f$ : $S^{1} \times[0,1] \rightarrow M$ be a map such that $f\left(S^{1} \times 0\right)=l, f\left(S^{1} \times 1\right)=k_{1}$, and $f$ is transverse with respect to $C$. Since $l$ is not contractible, the simple closed curve components of $f^{-1}(C)$ can be removed by a simple cutting and pasting operation. Further, $l \cap C=\varnothing$ so that no spanning arc in $f^{-1}(C)$ has an endpoint on $S^{1} \times 0$. Let $\beta$ denote a spanning arc in $f^{-1}(C)$ with endpoints on $S^{1} \times 1$. Then $\beta$ cobounds a disk with a subarc $\lambda$ of $S^{1} \times 1$. Since $C$ is irreducible with respect to $F, f(\lambda)$ is homotopic in $F \cup C$ with endpoints fixed to an arc $\eta$ in bd $C$. Observe then that either $\eta$ or its complementary arc in the boundary of $C$ must lie in $F$. We choose the arc that lies in $F$ and continue to call it $\eta$. Note also that $\eta^{-1} f(\lambda)$ is a loop in $F$ that contracts in $M$ and hence in $F$. It follows that $\eta$ is homotopic in $F$ with endpoints fixed to $f(\lambda)$. But then replacing the subpath $f(\lambda)$ of $k_{1}$ by $\eta$, a loop $k_{2} \in(k, F)$ is obtained with the property that $k_{2}$ is homotopic in $M$ to $l$, and $k_{2}$ meets $C$ fewer times than does $k_{1}$.

This contradiction assures us that in fact $k_{1} \cap C=\varnothing$, and the proof is complete.

2.4 LEMMA. Let $l$ be a simple closed curve in the boundary of a compact, orientable, irreducible 3-manifold $M$ with incompressible boundary. Let $K$ be an essential annulus in $M$ such that $K \cap l=\varnothing$. If $k$ is a loop in bd $M$ that is freely homotopic in $M$ to $l$, then there is a loop $k_{1} \in(k, \mathrm{bd} M)$ that is freely homotopic to $l$ via a homotopy of type 0,1 , or 2 with respect to $K$.

Proof. Let $F: S^{1} \times[0,1] \rightarrow M$ be a map such that $f\left|S^{1} \times 0=l, f\right| S^{1} \times 1$ $=k$, and $f$ is transverse with respect to $K$. Using the incompressibility of $K$ and bd $M$, the fact that $K$ is essential, and that $l \cap K=\varnothing$, it is easy to alter $f$ so that $f^{-1}(K)$ consists entirely of nontrivial simple closed curves $\gamma_{1}, \ldots, \gamma_{r}$ on $S^{1} \times[0,1]$. If $r \leqslant 1$, then $f$ is a type 0 or 1 homotopy as required. 
If $r>2$, then by a theorem of Jaco [5], it is true that $f\left(\gamma_{i}\right)$ is homotopic in $K$ to $f\left(\gamma_{1}\right)^{ \pm 1}$ for each $i(1 \leqslant i \leqslant r)$. Furthermore, if $f\left(\gamma_{i}\right)$ is homotopic in $K$ to $f\left(\gamma_{j}\right)$ then $\gamma_{i}$ and $\gamma_{j}$ can be removed from the preimage of $f^{-1}(K)$ by a simple cutting and pasting operation on $S^{1} \times[0,1]$. If as many of the $\gamma_{i}$ as possible are removed from $f^{-1}(K)$ by this process, then $f$ is clearly a type 0,1 , or 2 homotopy with respect to $K$.

2.5 Lemma. Let $M$ be a compact, orientable, irreducible 3-manifold with incompressible boundary. Let $K$ be an essential annulus in $M$ and $l$ a loop in bd $M$ that meets $K$ essentially at a point $P$. Let $\lambda$ be any path on $K$ joining $P$ to a point $q$ on the other boundary component of $K$. If there is a class ( $k, \mathrm{bd} M$ ) such that $l \notin(k$, bd $M)$ but $l$ is freely homotopic in $M$ to $k$, then $\lambda^{-1} l \lambda$ is homotopic with $q$ fixed to a loop in ( $k, \mathrm{bd} M)$. Furthermore, if such a class exists, it is unique.

Proof. Suppose $k$ is a loop in bd $M$ that is freely homotopic in $M$ to $l$, but $l \notin(k$, bd $M)$. In order to prove the theorem, it suffices to show that $\lambda^{-1} l \lambda$ is homotopic in $M$ with $q$ fixed to a member of $(k, \mathrm{bd} M)$. To this end, let $f$ : $S^{1} \times[0,1] \rightarrow M$ be a map such that $f\left|S^{1} \times 0=l, f\right| S^{1} \times 1=k, f(1,0)=$ $P$, and $f$ is transverse with respect to $K$. The component $\alpha$ of $f^{-1}(K)$ that meets $(1,0)$ is a spanning arc on $S^{1} \times[0,1]$. Since $P$ is an essential intersection point, $\alpha$ must have 1 endpoint on each boundary component of $S^{1} \times$ $[0,1]$. With no loss of generality $(1,1)$ is taken to be an endpoint of $\alpha$. There are two cases to be considered.

Case 1. $f(1,1)$ lies on the same boundary component of $K$ as $q$. The map $f$ can be corrected along bd $K$ so that $f(1,1)=q$. Observe that the loop $f(\alpha)^{-1} \lambda$ is homotopic with $q$ fixed to a loop $\sigma$ in bd $K$. In particular, $\sigma^{-1} k \sigma \in(k$, bd $M)$. Let $f_{1}: S^{1} \times[1,2] \rightarrow M$ be a map such that $f_{1} \mid S^{1} \times 1=$ $k, f_{1} \mid S^{1} \times 2=\sigma^{-1} k \sigma$ and $f_{1} \mid 1 \times[1,2]=f(\alpha)^{-1} \lambda$.

The maps $f$ and $f_{1}$ can be pasted together at the $S^{1} \times 1$ level to produce a map $g: S^{1} \times[0,2] \rightarrow M$. Observe that $g \mid \alpha \cup 1 \times[1,2]$ is homotopic with endpoints fixed to $\lambda$. Hence $\lambda^{-1} l \lambda$ is homotopic with $q$ fixed to $\sigma^{-1} k \sigma \in$ $(k$, bd $M)$.

Case 2. $f(1,1)$ lies in the boundary component of $K$ containing $P$. An argument similar to that used in Case 1 shows that $l$ is homotopic in $M$ with $P$ fixed to a loop $k_{1} \in(k, \mathrm{bd} M)$. But bd $M$ is incompressible so that in fact $l$ is homotopic to $k_{1}$ in bd $M$. Thus $l \in(k, \mathrm{bd} M)$ contrary to our assumption.

2.6 LemMa. Let $M$ be a compact, orientable, irreducible 3-manifold and $F$ an incompressible subset of bd $M$. Let $D$ be an essential disk in $M$ and $l a$ loop in bd $M$ that meets $D$ essentially at a point $P$. Let $R$ be a component of $F \cap D$ and $\lambda$ a path in $D$ joining $P$ to a point $q$ in $R$. There is a class $(k, F)$ that is freely homotopic in $M$ to $l$ such that some element of $(k, F)$ meets $R$ essentially 
only if $\lambda^{-1} l \lambda$ is homotopic with $q$ fixed to a loop in $F$. Furthermore, if this class exists, it is unique.

The proof is similar to the argument in Lemma 2.5 (but less complex) and is omitted.

Two other known algorithms are required for our constructions.

TheOREM 2.7 (HAKEN [4]). Let $M$ be a compact, orientable, irreducible 3-manifold. For any value of $\chi$, there is an algorithm $H$ for finding out if there exists in $M$ a cutting surface $F$ with $\chi(F)>\chi$. If such a surface exists, the algorithm will find one.

THEOREM 2.8 (WALDHAUSEN [7]). Let $M$ be a compact, orientable, irreducible 3-manifold and $F$ an incompressible subset of bd $M$. There is an algorithm $W$ for determining if any path $k$ with endpoints in $F$ is homotopic in $M$ with endpoints fixed to a path in $F$.

Finally we shall require involving free homotopies in 2-manifolds. The proofs of the next two lemmas are straightforward (especially in view of the fact that the conjugacy problem is solved for 2-manifold groups [1]) and are omitted.

2.9 LEMMA. There is an algorithm $S$ to determine if two loops in a compact, orientable 2-manifold are freely homotopic.

2.10 LEMMA. Let $G$ be a compact, orientable 2-manifold and $F$ an incompressible submanifold. There is an algorithm SA that will construct for any loop l in $G$ a representative from each class $(k, F)$ whose members are freely homotopic in $G$ to $l$.

3. The algorithms. In [4], Haken observes that with each compact, orientable, irreducible 3-manifold with boundary $M$, there is associated an integer $n(M)$ with the property that the result of cutting $M$ along any sequence of $n(M)$ non-disk-cutting surfaces is a cube-with-handles. (The reader is cautioned that this is a stronger theorem that Waldhausen's more often quoted Hierarchy theorem [8].) We shall refer to the number $n(M)$ as the length of $M$.

3.1 THEOREM. There is an algorithm BA that will construct for any loop l in the boundary of a compact, orientable, irreducible, sufficiently large 3-manifold $M$ and for any incompressible submanifold $F$ of bd $M$ one representative from each class $(k, F)$ such that $l$ is freely homotopic in $M$ to $k$.

Proof. The algorithm BA will in fact be presented as a series of algorithms. In particular, the algorithm BA $n$ is the algorithm BA for 3-manifolds of length no greater than $n$. BA $n, 0$ (resp. BA $n, 1$ ) refers to 3-manifolds of length no greater than $n$ that are known to contain no essential disks (resp. 
that are known to contain at least one essential disk). The algorithm BA 0, 0 is, of course, trivial. Waldhausen's solution of the word problem [7] is applied to $l$ to determine if $l$ is contractible in $M$. If $l$ is contractible, then 1 point in each component of $F$ will suffice for the classes $(k, F)$. In what follows, $l$ is taken to be noncontractible.

Step 1. Given BA $n-1$, the algorithm BA $n, 0$ can be constructed. At this stage the boundary of $M$ is by assumption incompressible. We shall obtain those classes $(k, \mathrm{bd} M)$ such that $l$ is freely homotopic in $M$ to $k$. The SA algorithm is then available to complete this step.

Haken's algorithm is first applied to construct a maximal Euler characteristic cutting surface $K$ for $M$. If $\chi(K)<0$, then, according to a theorem of Waldhausen [7], each free homotopy of $l$ with a loop $k$ in bd $M$ can be deformed into bd $M$. The loop $l$ itself is taken as the required representative.

Suppose then that $K$ is an essential annulus. By repeated application of Waldhausen's algorithm W, the number of points in $l \cap K$ can be minimized. Let $N$ denote $M$ cut at $K$ and $K_{0}, K_{1}$ annuli in the boundary of $N$ that are identified to obtain $M$.

Case 1. $K \cap l=\varnothing$. Then according to Lemma 2.4, only homotopies of type 0,1 , or 2 need be considered. The algorithm BA $n-1$ is available to construct homotopies of type 0 . In order to construct possible homotopies of types 1 and 2, BA $n-1$ is applied to produce representatives $\alpha_{1}, \ldots, \alpha_{r}$ for the classes $\left(k, K_{0} \cup K_{1}\right)$ that are freely homotopic in $N$ to $l$. Appealing once more to a theorem of Jaco [5], we see that $r<4$. If $r=0$, then all homotopies must be of type 0 and these have already been constructed.

If $r=1$, then say $\alpha_{1} \subset K_{0}$ and $\alpha_{1}$ is identified in $M$ with a loop $\beta_{1}$ in $K_{1}$. Type 1 homotopies in $M$ can now be constructed by applying BA $n-1$ to $N$ to find representatives for those classes $(k$, (bd $M) \cap N)$ that are freely homotopic in $N$ to $\beta_{1}$. Type 2 homotopies may also occur. Now $\beta_{1}$ is not freely homotopic in $N$ to $\alpha_{1}$ or to $\alpha_{1}^{-1}$ since $\beta_{1}$ is not freely homotopic in $N$ to $l$ or to $l^{-1}$; however, $\beta_{1}$ may be homotopic in $N$ to $\beta_{1}^{-1}$. (This can be determined by applying BA $n-1$.) If $\beta_{1}$ is not freely homotopic in $N$ to $\beta_{1}^{-1}$, then no type 2 homotopies occur, and the required classes have been constructed. If $\beta_{1}$ is freely homotopic in $N$ to $\beta_{1}^{-1}$, then the type 2 homotopies can be constructed by adding the inverse of each representative in (bd $M) \cap N$ that occurs as a type 0 homotopy.

For $r=2$, the situation becomes a bit more complex. If $\alpha_{1}$ and $\alpha_{2}$ both lie on $K_{0}$, then $\alpha_{1}=\alpha_{2}^{-1}$ on $K_{0}$ (again by [5]). Let $\alpha_{1}$ be identified with the loop $\beta_{1}$ on $K_{1}$ in $M$. Type 1 homotopies are then constructed by appealing to BA $n-1$ to produce the classes $\left(k_{1},(\right.$ bd $\left.M) \cap N\right)$ that are freely homotopic in $N$ to $\beta_{1}$ and the classes $\left(k_{1},(\mathrm{bd} M) \cap N\right)$ that are freely homotopic in $N$ to $\beta_{1}^{-1}$. Type 2 homotopies can in this case always be replaced by type 0 
homotopies since $\alpha_{1}$ is freely homotopic in $N$ to $\alpha_{1}^{-1}=\alpha_{2}$. The second possibility is that $\alpha_{1} \subset K_{0}$ and $\alpha_{2} \subset K_{1}$. Let $\alpha_{1}$ be identified with $\beta_{1} \subset K_{1}$ and $\alpha_{2}$ identified with $\beta_{2} \subset K_{0}$ in $M$. Type 1 homotopies can be produced by using BA $n-1$ to construct the classes $\left(k_{1},(\mathrm{bd} M) \cap N\right)$ that are freely homotopic in $N$ to $\beta_{1}$, and the classes $\left(k_{2},(\mathrm{bd} M) \cap N\right)$ that are freely homotopic in $N$ to $\beta_{2}$. Now $\beta_{1}$ may be homotopic in $N$ to $\beta_{1}^{-1}$ in which case type 2 homotopies occur as elements of $(k,(\mathrm{bd} M) \cap N)$ that are freely homotopic in $N$ to $\alpha_{1}^{-1}$. A second possibility is that $\beta_{1}$ is freely homotopic in $N$ to $\alpha_{1}^{-1}$. In this case, we obtain still more type 2 homotopies by adding the classes $(k$, (bd $M) \cap N)$ that are freely homotopic in $N$ to $\beta_{1}^{-1}$. A similar analysis must be carried out for $\beta_{2}$.

The value $r=3$ is not possible, for if $r=3$, then say $\alpha_{1} \in K_{0}, \alpha_{2}, \alpha_{3} \in K_{1}$. Since $\alpha_{2}$ is freely homotopic in $N$ to $\alpha_{3}$, it must be that $\alpha_{3}=\alpha_{2}^{-1}$ in $K_{1}$. But $\alpha_{2}$ is freely homotopic in $N$ to $\alpha_{1}$ so that $\alpha_{1}^{-1}$ is freely homotopic in $N$ to $\alpha_{2}^{-1}=\alpha_{3}$ and hence to $l$. Hence if $r>3$ then in fact $r=4$.

For $r=4$, let $\alpha_{1}, \alpha_{2} \in K_{0}$ and $\alpha_{3}, \alpha_{4} \in K_{1}$. Then $\alpha_{1}=\alpha_{2}^{-1}$ and $\alpha_{3}=\alpha_{4}^{-1}$. It follows immediately that type 2 homotopies can always be replaced by type 0 homotopies. The construction of type 1 homotopies is similar to constructions in earlier cases and is left to the reader.

Case 2. $l \cap K \neq \varnothing$. In this case we are requred to construct type 3 homotopies of $l$. Let $P$ be a point of $l \cap K$ and choose an $\operatorname{arc} \lambda$ on $K$ joining $P$ to a point $q$ on the opposite boundary component of $K$. Waldhausen's algorithm W can be applied to determine if $\lambda^{-1} l \lambda$ is homotopic in $M$ with $q$ fixed to a loop in bd $M$. According to Lemma 2.5, this is sufficient to determine all classes $(k, \mathrm{bd} M)$ such that $l$ is freely homotopic in $M$ to $k$. This completes the proof of Step 1.

Step 2. Given BA $n, 0$, it is possible to construct BA $n, 1$.

Proof. By assumption, $M$ contains an essential disk. The algorithm produced in Lemma 2.2 is applied to produce a complete set of cutting disks $C$ that is irreducible with respect to $F$. Waldhausen's algorithm $W$ is then applied to minimize the number of points in $l \cap C$.

Case $1 . l \cap C=\varnothing$. Then $l$ lies in a component $N$ of $M$ cut at $\cup D_{i}$. According to Lemma 2.3, it suffices to consider type 0 homotopies with respect to $\cup D_{i}$. Since bd $N$ is incompressible, BA $n, 0$ is available to construct these homotopies.

Case 2. $l \cap D_{1} \neq \varnothing$. Let $D_{1} \in C$ and $P$ a point on $D_{1} \cap l$, and let $R_{1}, \ldots, R_{r}$ denote the components of $F \cap D_{1}$. Choose arcs $\lambda_{1}, \ldots, \lambda_{r}$ on $D_{1}$ joining $P$ to points $q_{1} \in R_{1}, \ldots, q_{r} \in R_{r}$. For each $i$ apply Waldhausen's algorithm W to determine if $\lambda_{i}^{-1} l \lambda_{i}$ is homotopic with $q$ fixed to a loop in $F$. According to Lemma 2.6, this process will produce the proper classes in $F$. This procedure is repeated for each $D_{i}$ such that $l \cap D_{i} \neq \varnothing$. This completes Step 2. 
At this point, Steps 1 and 2 together with Haken's algorithm $\mathrm{H}$ establishes the existence of the BA $n$ algorithm for each $n$. All that remains to establish BA is a procedure for determining an upper bound for the length of a 3-manifold given in terms of a triangulation. Haken notes in [4] that the length of $M$ is bounded above by the maximum number of disjoint, mutually nonparallel, closed incompressible 2-manifolds that can be embedded in $M$, and that this number is in turn bounded above by a number that can be computed from a triangulation of $M$ [3]. This completes the proof of Theorem 3.1.

3.2 THEOREM. There is an algorithm A that will determine for any pair of loops $l_{1}$ and $l_{2}$ in the boundary of a compact, orientable, irreducible, sufficiently large 3-manifold $M$ if $l_{1}$ is freely homotopic in $M$ to $l_{2}$.

Proof. Haken's algorithm is first applied to determine if $M$ contains an essential disk.

Case 1. $M$ contains no essential disk. The algorithm BA can be applied to construct those classes $\left(k_{1}\right.$, bd $\left.M\right), \ldots,\left(k_{r}\right.$, bd $\left.M\right)$ such that $l_{1}$ is freely homotopic in $M$ to each $k_{i}$. Then the conjugacy problem for 2-manifolds can be applied to determine if $l_{2}$ lies in any of the classes $\left(k_{1}\right.$, bd $\left.M\right), \ldots,\left(k_{r}\right.$, bd $\left.M\right)$.

Case 2. $M$ contains an essential disk. Lemma 2.2 is applied to produce a complete set of cutting disks $C$ for $M$. Waldhausen's algorithm $\mathrm{W}$ is then applied to minimize the intersections of $l_{1} \cup l_{2}$ with $C$. If $l_{1}$ does not meet some disk $D_{i} \in C$ in exactly the same number of points as does $l_{2}$ then $l_{1}$ is not freely homotopic in $M$ to $l_{2}$. If $l_{1} \cap C=\varnothing$, then determination of conjugacy can be made as in Case 1 above. Suppose then that $P$ is a point of $l_{1} \cap D_{1}$, and let $l_{2} \cap D_{1}=\left\{q_{1}, \ldots, q_{s}\right\}$. For each $i$, let $\lambda_{i}$ be an arc in $D_{1}$ joining $P$ to $q_{i}$. Then $l_{1}$ is freely homotopic to $l_{2}$ in $M$ if and only if one of the loops $\lambda_{i} l_{2} \lambda_{i}^{-1} l_{1}^{-1}$ is contractible in $M$. Waldhausen's word problem algorithm [6] is available to determine the contractibility of these curves.

\section{REFERENCES}

1. M. Dehn, Über unendliche diskontinvierliche Gruppen, Math. Ann. 71 (1911), 116-144.

2. C. F. Feustel, The torus theorem and its applications (preprint).

3. W. Haken, Some results on surfaces in 3-manifolds, Studies in Modern Topology, Math. Assoc. Amer. (distributed by Prentice-Hall, Englewood Cliffs, N. J.), 1968, pp. 39-98. MR 36 \#7118.

4. 89-120. MR 28 \#3410.

5. W. Jaco, Roots, relations, and centralizers in three-manifold groups, Proc. Geometrical Topology Conf. (Utah, 1974), Lecture Notes in Math., vol 438, Springer-Verlag, Berlin and New York, 1975, pp. 283-309. MR 50 \# 14751.

6. F. Waldhausen, The word problem in fundamental groups of sufficiently large irreducible 
3-manifolds, Ann. of Math. (2) 88 (1968), 272-280. MR 39 \#2164.

7. On the determination of some 3-manifolds by their fundamental groups alone, Proc. Internat. Sympos. on Topology and Its Applications (Herceg-Novi, August 1968, Yugoslavia), Savez Društava Mat., Fix. i Astronom., Belgrade, 1969, pp. 331-332. MR 42 \#2416.

8. On irreducible 3-manifolds which are sufficiently large, Ann. of Math. (2) 87 (1968), 56-88. MR 36 \#7146.

Department of Mathematics, Orlahoma State University, Stillwater, Orzahoma 74074 